descriptive study. An inclusive approach would seem particularly appropriate for those with severe handicap where the aetiology of repetitive movements is speculative.

Therefore the descriptive categories of the DISCo or DISCUS can be appropriately employed, provided that attention is adequately paid to cooperation levels and their influence on study results. It must also be recognised that other movements, while not regarded as forming part of a tardive dyskinesia spectrum, represent aspects of motor disorder and are relevant. On this basis, a Nottingham group is currently examining abnormal movements in people with severe impairments.

Granger, D. A., Yurkunski, J. M., Miller, N. H., et al (1987) Systematic dyskinesia examination of profoundly mentally retarded persons: co-operation and assessment. American Journal of Mental Deficiency, 92, 155-160.

Sprague, R. L., KAlachini,, J. E., Breuning, S. E., et al (1984) The dyskinesia identification system-Coldwater (DIS-Co): A tardive dyskinesia rating scale for the developmentally disabled. Psychopharmacology Bulletin, 20, 328-338.

- — SHAw, K. M. (1989) Psychometric properties of the dyskinesia identification system: condensed user scale (DISCUS). Mental Retardation, 27, 141-148.

Department of Mental Handicap

JO JONES

Highbury Hospital

Bulwell

Nottingham NG6 9DR

\section{Delusional jealousy in paranoid disorders}

SIR: Soyka et al (Journal, April 1991, 158, 549-553) examined the prevalence of delusional jealousy in various psychiatric disorders and reported $6.7 \%$ in paranoid disorders. This might be an underestimate because of the low admission rates of patients in whom delusional jealousy is the only symptom of their illness, unlike patients with other psychotic syndromes. The authors themselves had highlighted the reluctance on the part of patients, and their spouses, to talk about this particular symptom, sometimes leading to difficulty in diagnosis. One would naturally expect more reluctance in treatment acceptance, especially when it comes to admission to psychiatric hospital. Thus by studying only in-patients, the authors could not exclude the possibility of selection bias affecting the prevalence of delusional jealousy.

We reviewed the case records of 297 patients diagnosed to have a paranoid disorder (ICD-9; World Health Organization, 1978) who presented to our department over a 10-year period (1979-1989). Of the 93 patients who received a diagnosis of a paranoid disorder, delusional jealousy was documented in 15 individuals, giving the symptom a prevalence of $16 \%$. Among the 16 patients with paranoid disorders who received in-patient treatment, only one individual had delusions of jealousy. In our series, the prevalence of this symptom among in-patients is similar to that of Soyka et al. Thus, our findings show marked differences in the prevalence of the symptom dependent on the admission status, with a higher figure for out-patients than among those admitted to the wards.

When DSM-III-R criteria were applied to these case records, only 60 individuals met the criteria for delusional disorder. Interestingly, all the patients with delusions of jealousy met the criteria, thus increasing the prevalence of delusional jealousy to $25 \%$ among patients with delusional disorder. This suggests that the actual prevalence of delusional jealousy could be higher with the use of more restrictive criteria. However, the prevalence of this symptom in the community would probably be different and would be difficult to document.

World Health Organization (1978) Mental Disorders: Glossary and Guide to their Classification in accordance with the minth revision of the International Classification of Diseases (ICD-9). Geneva: WHO.

Department of Psychiatry

K. S. SHAJ

Christian Medical College

Vellore 632002

India

SIR: We read with interest Soyka et al's study (Journal, April 1991, 158, 549-553) of the prevalence of delusional jealousy, and would like to offer the following comments.

Firstly, delusional jealousy involves the lover, marital or sexual partner and hence occurs in those who have or have ever had one. Therefore, considering the whole patient population, ignoring the marital or sexual status and history, and including those who never had a lover or partner, as done in this study, would give erroneously low figures.

Secondly, the authors suggest that the difference in prevalence of delusional jealousy in affective disorders $(0.1 \%)$ and schizophrenia $(2.5 \%)$ may be of value in the differential diagnosis of the two disorders. This opinion is rather far fetched in view of the fact that among those with delusional jealousy $3-16 \%$ had depressive illness, while $17-44 \%$ had schizophrenia (Gelder et al, 1989).

Finally, this study supports the surprising earlier observations (Gelder et al, 1989) of 'delusional' jealousy occurring in 'neuroses'. 